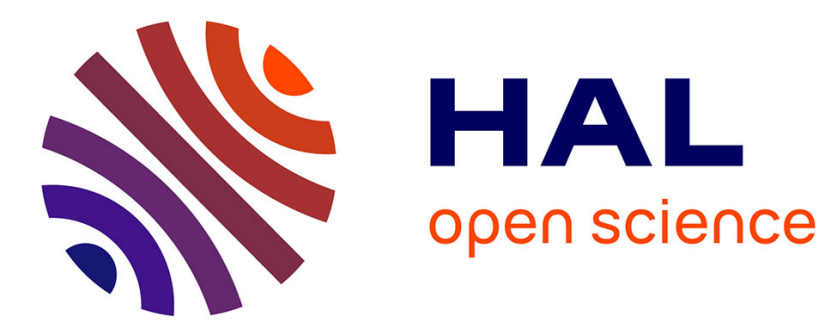

\title{
Using Relative Neighborhood Graphs for Reliable Database Synchronization in MANETs
}

\author{
Emmanuel Baccelli, Juan Antonio Cordero Fuertes, Philippe Jacquet
}

\section{To cite this version:}

Emmanuel Baccelli, Juan Antonio Cordero Fuertes, Philippe Jacquet. Using Relative Neighborhood Graphs for Reliable Database Synchronization in MANETs. WIMESH 2010 - Fifth IEEE Workshop On Wireless Mesh Networks, Jun 2010, Boston, United States. pp.1 - 6, 10.1109/WIMESH.2010.5507907 . hal-00651590

\section{HAL Id: hal-00651590 \\ https://hal.science/hal-00651590}

Submitted on 13 Dec 2011

HAL is a multi-disciplinary open access archive for the deposit and dissemination of scientific research documents, whether they are published or not. The documents may come from teaching and research institutions in France or abroad, or from public or private research centers.
L'archive ouverte pluridisciplinaire HAL, est destinée au dépôt et à la diffusion de documents scientifiques de niveau recherche, publiés ou non, émanant des établissements d'enseignement et de recherche français ou étrangers, des laboratoires publics ou privés. 


\title{
Using Relative Neighborhood Graphs for Reliable Database Synchronization in MANETs
}

\author{
Emmanuel Baccelli, Juan Antonio Cordero and Philippe Jacquet \\ E-mails: Emmanuel.Baccelli@inria.fr, cordero@lix.polytechnique.fr, Philippe.Jacquet@inria.fr \\ INRIA Saclay - Île-de-France \\ Parc Orsay Université, 4 rue J. Monod, 91893 Orsay Cedex, France
}

\begin{abstract}
Achieving reliable communication of critical data on mobile ad hoc networks is a must before MANETs can be considered practical for standard mobile and vehicular communications. This paper thus focuses on schemes that complement existing ad hoc broadcast mechanisms (inherently unreliable), which can guarantee the full diffusion of critical messages, when necessary. An interesting approach in this domain is the use of an overlay network, over which critical messages are acknowledged peer to peer, in order to verify the actual dissemination. This paper elaborates on the problem of performance, related to the discovery and the maintenance of such an overlay network, in a mobile ad hoc context. We present an analysis of a novel algorithm, SLOT (Synchronized Link Overlay - Triangular), an overlay selected based on a Relative Neighborhood Graph (RNG) scheme, and we compare its performance with that of other existing algorithms, in the context of the industry-standard IP routing protocol OSPF, which uses alternative overlay algorithms to synchronize link state databases of routers. The results presented in this paper show that SLOT outperforms other existing overlays by necessitating drastically less control traffic in order to function, enabling OSPF to scale to larger mobile ad hoc topologies.
\end{abstract}

\section{INTRODUCTION}

While MANET routing has been the subject of extensive research and experiments in the recent years, reliable communication of critical data over mobile ad hoc networks is still a challenge, especially as the number of nodes in the network grows large. Achieving such reliability is however necessary before mobile ad hoc networks can be considered practical for standard mobile and vehicular communications. This paper thus focuses on schemes that are complementary to existing ad hoc broadcast mechanisms, which guarantee the full diffusion of critical messages, when necessary. One interesting approach in this realm is the use of an overlay network, i.e. a subgraph over which critical messages are acknowledged hop-by-hop, in a peer to peer fashion, in order to verify the actual dissemination, or, absent expected acknowledgement, retransmit the corresponding critical message. Such an approach ensures that the respective databases of overlay nodes remain synchronized with one another over time.

This paper elaborates on the problem of performance, related to the discovery and the maintenance of such an overlay network, in a mobile ad hoc context. Related work in this domain mainly include connected dominating set schemes such as MPR [4] and CDS schemes such as [26] and [28]. This paper analyzes the theoretical performance of SLOT (Synchronized Link Overlay - Triangular), an overlay selected based on a Relative Neighborhood Graph (RNG) scheme described in the following. SLOT operation is defined in two modes: cost-based and ID-based. These two versions of the SLOT scheme are analyzed and compared in terms of link set size and stability.

The paper then applies SLOT to the industry-standard IP routing protocol OSPF [1][2], which relies on such a local database synchronization scheme for critical control data. Simulations presented in this paper show that the use of SLOT in this context drastically reduces the amount of control traffic overhead, and allows OSPF to scale to much larger ad hoc topologies compared to similar existing solutions (RFC 5449 [10], and RFC 5614 [27]).

\section{THE SLOT ALGORITHM}

The goal of this section is to analyze the performance of a scheme selecting an overlay network in a mobile ad hoc context. This overlay network is to be used to synchronize critical data among the different nodes in the ad hoc network.

\section{A. Requirements}

Since the mobility of these nodes can be substantial and ad hoc communication resources are generally scarce, it is essential to select an overlay network that fits best the following targets:

1) Low overlay link density;

2) Low overlay link change rates.

Low overlay link density is paramount because a high density results in a huge number of acknowledgement and data packets (re)transmissions. For example, let's consider a high density network with $N$ nodes, having each $M$ neighbor, on average. If the overlay network were the full network, then the transmission of a single critical message will generate a quadratic $N M$ number of acknowledgements and packet transmissions. Experiments with standard OSPF show that wifi based networks cannot support more than 20 nodes [19] without collapsing due to packet retransmissions and 
acknowledgement cascade, if the overlay network is the whole network.

Low overlay link change rate is a critical consequence of node mobility and wireless medium variations. It is however not necessarily a simple function of the overlay link density. As we will see in this paper, it is possible to have an overlay $S_{1}$ with a lower overlay link density than another overlay $S_{2}$, while the latter generates less overlay link changes. Nevertheless, if the overlay network is the whole network and if the average link lifetime is $T$, then the new overlay link creation rate will be $\frac{M N}{T}$, which is the rate of basic peer to peer database synchronization.

In this section we introduce a scheme, SLOT, which identifies and maintains an overlay network, based on Relative Neighbor Graph (RNG [16] [23], itself inspired from the Gabriel graph [15], except RNG does not need positioning devices, only link cost estimates). A subset of the whole set of links network-wide, are selected to be part of the overlay network. Links can be selected according to link quality, in order to reduce the packet loss and the cost of data synchronization. It is indeed anticipated that future radio networks will operate on on-line programmable radio-interface which will naturally increase the throughput when nodes are closer. In this case the cost metric can be a function increasing with the distance.

We will first specify the SLOT scheme, and then evaluate it analytically with a model based on unit disk graph and random walk mobility in two dimensions. We will then generalize these results for other dimensions.

\section{B. Algorithm Details}

Since synchronization happens symmetrically on overlay links, these links must of course be bidirectional. In this context, if $m(A, B)$ is the metric on the symmetric link $(A, B)$, we then have $m(A, B)=m(B, A)$. Let's for instance take $m(A, B)=\min \{\operatorname{Cost}(A, B), \operatorname{Cost}(B, A)$, where $\operatorname{Cost}()$. is a specific metric (such as bit delivery delay, remaining bandwidth, financial cost).

A symmetric link $(A, B)$ belongs to the overlay except if there exists a chain of links $\left(A, C_{1}\right), \ldots,\left(C_{i}, C_{i+1}\right), \ldots$, $\left(C_{k}, B\right)$ such that:

- The $C_{i}$ are all neighbors of both $A$ and $B$.

- The cost of the links,
$m\left(A, C_{1}\right), \ldots, m\left(C_{i}, C_{i+1}\right), \ldots, m\left(C_{k}, B\right), \quad$ are all
smaller to $m(A, B)$.

In case of link cost equality, the following tie breaking is used: the link with lower cost is the link with the minimum node ID. In case this rule still does not tie the break (because the two links have their minimum ID node in common), then the considered links are part of the overlay.

SLOT is defined as a simplification of the above, considering only triangular relations: a link $(A, B)$ is not selected as part of the overlay if and only if there exist a common neighbor node $C$ such that $m(A, B) \geq \max \{m(A, C), m(C, B)\}$. This can be used when the metric satisfies the triangular inequality: $m(A, B) \leq m(A, C)+m(C, B)$.

When the link have all the same (uniform) cost, in this case a link $(A, B)$ is removed from the overlay when:

- there exist a node $C$ neighbor to both $A$ and $B$;

- node $C$ ID is smaller to both node $A$ and node $B$ ID's.

In this case the elimination is equivalent to a triangle elimination: the link holding the two largest node ID is eliminated. Fig. 1 shows two examples of SLOT overlay, one with cost based on distance and the second with uniform cost, compared to the total number of network links, with the unit disk graph model.

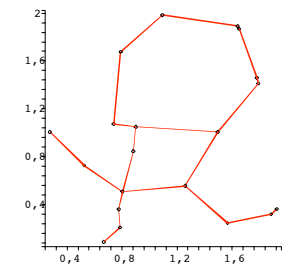

(a) SLOT with link cost based on distance.

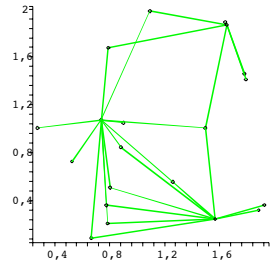

(b) SLOT based on IDs (uniform link cost).

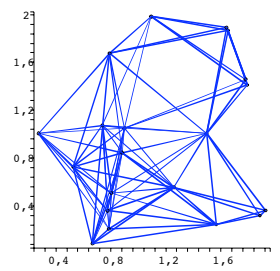

(c) Full network.

Fig. 1. Overlay links, for a network with 20 nodes on a $2 \times 2$ map.

\section{ANALYSIS OF SLOT IN DIMENSION 2}

This section provides a theoretical performance of the SLOT algorithm. In the following we consider the unit-disk graph model of uniform node density $\nu$ and a plan of area $\mathcal{A}$. Therefore the average node neighborhood size is $M_{f}=\pi \nu$. We assume that each node follows an independent random walk equivalent to an average speed of $s$. We also assume that the random walk is isotropic, so that the stationary probability that a node is in a portion of the map of area $\sigma$ and has speed in a cone of aperture $\theta$ is exactly $\frac{\theta \sigma}{2 \pi \mathcal{A}}$. We will consider the asymptotic case where $\nu \rightarrow \infty$ and $\mathcal{A} \rightarrow \infty$. In a first phase we will analyze the performance of SLOT in a two dimension vector map. In a second phase we generalize to other dimensions $D$ : a linear map $(D=1)$ and a cubic map $(D=3)$. 


\section{A. General Properties}

Let's observe that the mobility model is sufficiently general. We assume that the nodes move at a constant speed $s$ on a random distance $L$ and then change direction. When $L$ is large, assume $L=\infty$ on an infinite map, this is equivalent to a kind of random waypoint mobility model. When $L \rightarrow 0$ then it is equivalent to a Brownian motion model. However all the independent random walk models have in common the following properties [18].

1) The number of points in a portion of map of size $b$ is a Poisson distribution of density $b \nu$.

2) The rate $f(B)$ at which nodes enter the portion $B$ is proportional to the node density, the length $\ell$ of the border and the average speed of the mobile, that is $f(B)=\frac{\ell s}{\pi} \nu$.

3) If we consider that the portion of the map $B$ also moves like a mobile node, then the rate at which mobile nodes enter the mobile area becomes $\frac{\ell \Delta(s)}{\pi} \nu$ where $\Delta(s)$ is the average relative speed between two random mobile nodes.

Note that if the nodes move at constant speed $s$ (no randomness), then we get $\Delta(s)=\frac{4}{\pi} s$, otherwise the formula is more complicated.

\section{B. Full Network Overlay}

In this section we investigate the specific case where the overlay network is in fact the whole network. The rate at which nodes enter a node neighborhood is $V_{f}=2 \Delta(s) \nu$. We have thus proven the following theorem:

Theorem 1: The average number of links departing from a node is equal to $M_{f}=\pi \nu$ and the average rate of new links per second is equal to $V_{f}=2 \Delta(s) \nu$. If nodes move at the same speed $s$ we have $V_{f}=\frac{8}{\pi} s \nu$.

\section{SLOT Based on Distance}

In this section we investigate the case where the overlay is determined by the SLOT algorithm based on the distance cost metric. Let's begin with a straightforward theorem.

Theorem 2: The graph of the SLOT overlay based on distance is planar.

Proof: This is a well known property of RNG [16], if two links cross over, then it is easy to see that one at least will be eliminated in the SLOT algorithm.

Theorem 3: The average number of overlay links departing from a node in the SLOT algorithm with cost based on distance is equal to [17]

$$
M_{d}=\frac{\pi}{2 \frac{\pi}{3}-\frac{\sqrt{3}}{2}}+O\left(\nu e^{-\left(\frac{2 \pi}{3}-\frac{\sqrt{3}}{2}\right) \nu}\right)
$$

and the average rate of new synchronized links from a node is equal to

$$
\begin{aligned}
V_{d}= & \frac{4}{3 \sqrt{\pi}}\left(2 \frac{\pi}{3}-\frac{\sqrt{3}}{2}\right)^{-\frac{3}{2}} \Delta(s) \sqrt{\nu} \\
& +O\left(\Delta(s) \nu^{2} e^{-\left(\frac{2 \pi}{3}-\frac{\sqrt{3}}{2}\right) \nu}\right)
\end{aligned}
$$

Remark: For constant uniform speed $s$ it comes $V_{d}=$ $\sqrt{\pi} \frac{8}{3}\left(2 \frac{\pi}{3}-\frac{\sqrt{3}}{2}\right)^{-\frac{3}{2}} s \sqrt{\nu}$

Proof: Let's consider a link between two nodes $A$ and $B$ at distance $r$ of each other. Let $B(r)$ be the intersection of the disks of radius $r$ respectively centered on $A$ and $B$. The condition at which the link belongs to the overlay is that $B(r)$ contains no other nodes than $A$ and $B$. The area of this intersection is $|B(r)|=r^{2} A\left(\frac{\pi}{3}\right)$ with $A(\theta)=2 \theta-\sin (2 \theta)$. Therefore the probability that link $(A, B)$ is included in the overlay is $\exp \left(-\nu r^{2} A\left(\frac{\pi}{3}\right)\right)$.

The average number of links from a random node $A, M_{d}$, that belong to the overlay will be

$$
\begin{aligned}
M_{d}= & \int_{0}^{1} 2 \pi \nu r d r e^{-r^{2} A\left(\frac{\pi}{3}\right) \nu} \\
= & \int_{0}^{\infty} 2 \pi \nu r d r e^{-r^{2} A\left(\frac{\pi}{3}\right) \nu} \\
& +O\left(\nu e^{-\nu|B(1)|}\right) \\
= & \frac{\pi}{2 \frac{\pi}{3}-\frac{\sqrt{3}}{2}}+O\left(\nu e^{-\nu|B(1)|}\right) \\
\approx & 2.557530242+O\left(\nu e^{-\nu|B(1)|}\right)
\end{aligned}
$$

The constant $M_{d}$, Devroye's constant, is known from [17].

For the rate change, the computation is also relatively straightforward. Let's consider a link $(A, B)$ which belongs to the overlay. $B(r)$, the intersection of the corresponding disks of radius $r$, is empty. Therefore the rate at which the link will disappear from the overlay is equal to the rate at which the mobile nodes will enter $B(r)$. Let's call $\partial B(r)$ the border of $B(r)$ and its length $|\partial B(r)|=\frac{4}{3} \pi r$. The entering rate is therefore $|\partial B(r)| \frac{\Delta(s)}{\pi} \nu$. Therefore the rate $V_{d}$ at which overlay links vanish, from a random node $A$, is

$$
\begin{aligned}
V_{d}= & \frac{4}{3} \Delta(s) \int_{0}^{1} 2 \pi \nu^{2} r^{2} d r e^{-r^{2} A\left(\frac{\pi}{3}\right) \nu} \\
= & \frac{4}{3} \Delta(s) \int_{0}^{\infty} 2 \pi \nu^{2} r^{2} d r e^{-r^{2} A\left(\frac{\pi}{3}\right) \nu} \\
& +O\left(\nu^{2} e^{-\nu|B(1)|}\right) \\
= & \frac{4}{3 \sqrt{\pi}}\left(A\left(\frac{\pi}{3}\right)\right)^{-\frac{3}{2}} \Delta(s) \sqrt{\nu}+O\left(\nu^{2} e^{-\nu|B(1)|}\right)
\end{aligned}
$$

Notice that $V_{d} \approx 3.471762654 \times s \sqrt{\nu}$ when the speed is constant. The rate at which links appear is also $V_{d}$.

\section{SLOT with Uniform Link Cost}

In this section we investigate the case where the overlay is determined by the SLOT algorithm based on IDs (uniform link cost).

Theorem 4: The average number of overlay links departing from a node in the SLOT algorithm with uniform link cost, when $\nu \rightarrow \infty$ satisfies

$$
M_{u}=\int_{\frac{\pi}{3}}^{\frac{\pi}{2}} \frac{8 \pi \sin 2 \theta}{2 \theta-\sin 2 \theta} d \theta+O\left(\frac{1}{\nu}\right)
$$

with $A(\theta)=2 \theta-\sin 2 \theta$ and the average rate of new synchronized links from a node tends to

$$
V_{u}=32 \Delta(s) \int_{\frac{\pi}{3}}^{\frac{\pi}{2}} \frac{\theta \sin 2 \theta}{(2 \theta-\sin 2 \theta)^{2}} d \theta+O\left(\frac{1}{\nu}\right)
$$


Remark: When the speed is constant we have $\lim _{\nu \rightarrow \infty} V_{u}=\frac{128 s}{\pi} \int_{\frac{\pi}{3}}^{\frac{\pi}{2}} \frac{\theta \sin 2 \theta}{(2 \theta-\sin 2 \theta)^{2}} d \theta$.

Proof: Let's assume that node $A$ has ID equal to $x$, and $B$ has ID $y$. We consider that the link $(A, B)$ belongs to the overlay and $y>x$ (this will cover half the cases). Assume that the distance between node $A$ and node $B$ is $r$ and let $S(r)$ be the intersection of the disks of radius 1 , respectively centered on $A$ and $B$, i.e. the location of the common neighborhood of $A$ and $B$. Since $(A, B)$ is an overlay link there is no node in $S(r)$ with ID smaller than $\min \{x, y\}=x$.

Since we consider only ID comparison, there is no loss of generality to assume that the IDs of the nodes are scalar numbers uniformly distributed on the unit interval.

The probability that (i) $y>x$ and (ii) the link $(A, B)$ belongs to the overlay is $(1-x) \exp (-\nu x|S(r)|)$. Since $|S(r)|=A(\theta)$ with $r=2 \cos \theta$. Counting also the case where $y<x$ we get

$$
\begin{aligned}
M_{u}(\nu) & =2 \int_{0}^{1} d x \int_{0}^{1} 2 \pi \nu(1-x) r d r e^{-x A(\theta) \nu} \\
& =\int_{0}^{1} \int_{\frac{\pi}{3}}^{\frac{\pi}{2}} 8 \pi \nu(1-x) \sin 2 \theta d \theta e^{-x A(\theta) \nu} \\
& =\int_{\frac{\pi}{3}}^{\frac{\pi}{2}} \frac{8 \pi}{\nu(A(\theta))^{2}} \sin 2 \theta d \theta\left(\nu A(\theta)-1+e^{-\nu A(\theta)}\right)
\end{aligned}
$$

Therefore:

$$
\begin{aligned}
M_{u}(\nu) & =\int_{\frac{\pi}{3}}^{\frac{\pi}{2}} \frac{8 \pi}{A(\theta)} \sin 2 \theta d \theta+O\left(\frac{1}{\nu}\right) \\
& =M_{u}+O\left(\frac{1}{\nu}\right)
\end{aligned}
$$

with

$$
M_{u}=\int_{\frac{\pi}{3}}^{\frac{\pi}{2}} \frac{8 \pi \sin 2 \theta}{2 \theta-\sin 2 \theta} d \theta \approx 3.603973720
$$

To get the rate at which overlay links vanish in the uniform cost algorithm, we just have to consider the same link $(A, B)$ such that nodes are at distance $r$ and their IDs are respectively $x$ and $y$. We assume $x<y$. The area $S(r)$ contains no node with ID smaller than $x$. The rate at which the link $(A, B)$ will disappear as overlay link is equal to the rate at which nodes with ID smaller than $x$ will enter the area $S(r)$. This rate is equal to $|\partial S(r)| \frac{\Delta(s)}{\pi} \nu x$. Since $|\partial S(r)|=4 \theta$ with $r=2 \cos \theta$, the rate at which overlay links disappear (including the case $y<x$ ) is:

$$
\begin{aligned}
V_{u}(\nu)= & \Delta(s) \int_{0}^{1}(1-x) x d x \\
& \times \int_{\frac{\pi}{3}}^{\frac{\pi}{2}} 32 \nu^{2} \theta \sin 2 \theta d \theta e^{-\nu A(\theta) x} \\
= & \Delta(s) \int_{\frac{\pi}{3}}^{\frac{\pi}{2}} \frac{32 \theta \sin 2 \theta}{\nu(A(\theta))^{3}} \\
& \times\left(A(\theta) \nu-2+(2+\nu A(\theta)) e^{-\nu A(\theta)}\right) d \theta \\
= & V_{u}+O\left(\frac{\Delta(s)}{\nu}\right)
\end{aligned}
$$

with

$$
V_{u}=32 \Delta(s) \int_{\frac{\pi}{3}}^{\frac{\pi}{2}} \frac{\theta \sin 2 \theta}{(2 \theta-\sin 2 \theta)^{2}} d \theta
$$

When the speed is a constant $s$, we have $V_{u}=\frac{128 s}{\pi} \int_{\frac{\pi}{3}}^{\frac{\pi}{2}} \frac{\theta \sin 2 \theta}{(2 \theta-\sin 2 \theta)^{2}} d \theta \approx 4.146111863 \times s$

\section{ANALYSIS SUMmARY}

Table I summarizes our analysis of SLOT based on distance link cost, and of SLOT based on uniform link cost in dimension 2. An analysis for dimension 1 and dimension 3 is available in [29]. For SLOT based on distance link cost, the link density increases with dimension number, but the link change rate decreases. Clearly a generalization to higher dimension would show a link change rate in $O\left(\nu^{\frac{1}{D}}\right)$. With SLOT based on uniform link cost, on the other hand, density and change rates attain their maximum value for $D=2$ and then decrease. The qualitative explanation of this phenomenon is still an open problem.

\begin{tabular}{|r|ll|}
\hline & SLOT Distance \\
\hline$D$ & $M_{d}$ & $V_{d}$ \\
\hline 1 & 2 & $\nu \Delta(s) \times 2$ \\
\hline 2 & 2.557530242 & $\sqrt{\nu} \Delta(s) \times 2.726716013$ \\
\hline 3 & 2.944205854 & $\nu^{\frac{1}{3}} \Delta(s) \times 1.018993779$ \\
\hline \multicolumn{3}{|c|}{} \\
\hline \multicolumn{3}{|l|}{ SLOT Uniform } \\
\hline$D$ & $M_{u}$ & $V_{u}$ \\
\hline 1 & 2.772588722 & $\Delta(s) \times 2$ \\
\hline 2 & 3.603973720 & $\Delta(s) \times 3.603973720$ \\
\hline 3 & 2.5046958 & $\Delta(s) \times 1.441903356$ \\
\hline
\end{tabular}

TABLE I

RESULTS OF THE ANALYSIS OF SLOT BASED ON DISTANCE LINK COST (TOP TABLE) AND UNIFORM LINK COST (BOTTOM TABLE), FOR DIMENSIONS 1, 2 AND 3.

\section{SLOT OVERLAys APPLIED TO OSPF}

In the following, we will use the SLOT algorithm to provide a synchronization overlay for OSPF on ad hoc networks. OSPF is a proactive link state routing protocol, which uses a synchronization overlay to provide reliable link state database synchronization between routers in the network. So far, in a MANET environment, OSPF uses two connected dominating set (CDS) approaches to provide such an overlay: on one hand an approach based on MPR [4] specified in RFC 5449, and on the other hand an approach based on another CDS technique inspired from [26] specified in RFC 5614. For further details on OSPF, RFC 5449 or RFC 5614 see for example [25]. We now present simulation results, that compare the performance of RFC5449 and RFC 5614 with that of SLOT-OSPF, a refinement of RFC 5449 using SLOT synchronization overlays, instead of MPR.

Results shown below were obtained based on the Zebra OSPF implementation [13] running on $802.11 \mathrm{~b}$, simulated with the GTNetS [5] simulator. We used the random waypoint model at an average speed of $2,5 \mathrm{~m} / \mathrm{s}$ (in fact $5 \mathrm{~m} / \mathrm{s}$ with pauses of $40 \mathrm{sec}$ ), and we simulated CBR user data UDP flows that amount to a total of $1 \mathrm{Mbps}$, shared among all nodes. Implementation of RFC 5449 follows the IETF specification [10] and the RFC 5614 code is available in [24]. The code for SLOT-OSPF and for RFC 5449 is available [14]. See the appendix for more details on the simulations. 


\section{A. OSPF Simulations}
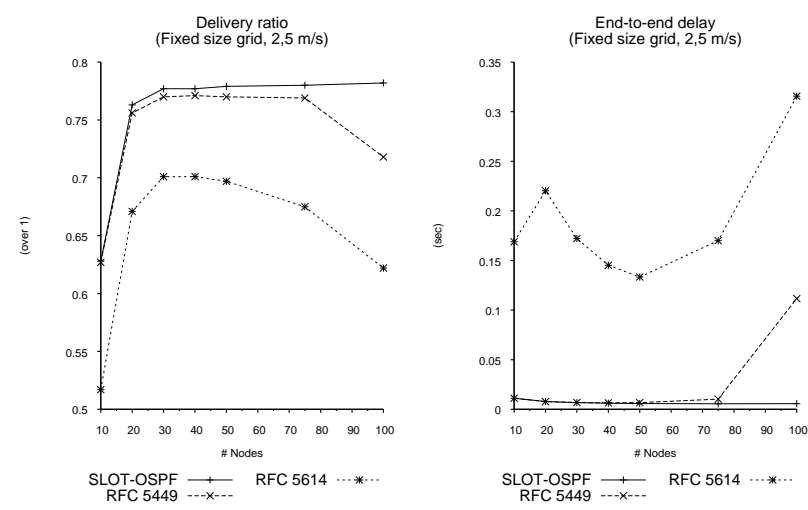

Fig. 2. Delivery ratio (left) and delay in seconds (right). CBR user data flows, $1 \mathrm{Mbps}$. Fixed size grid, 2,5 m/s velocity.
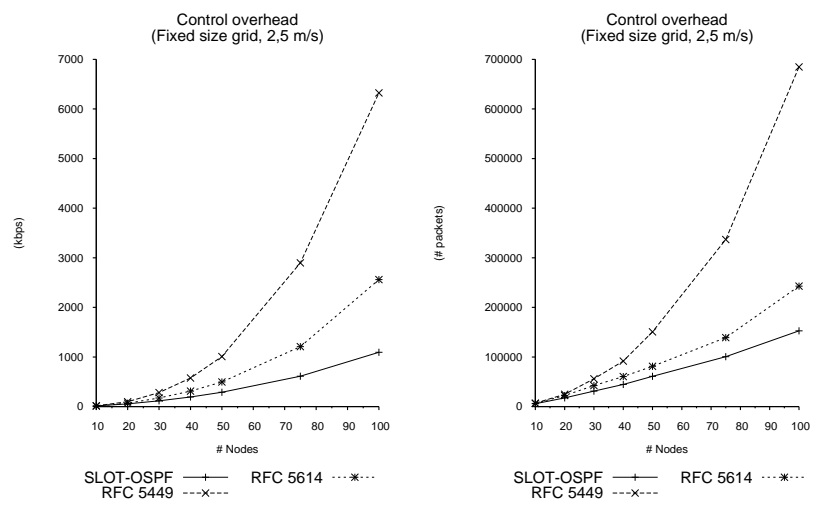

Fig. 3. Control overhead in kbps and in number of packets.
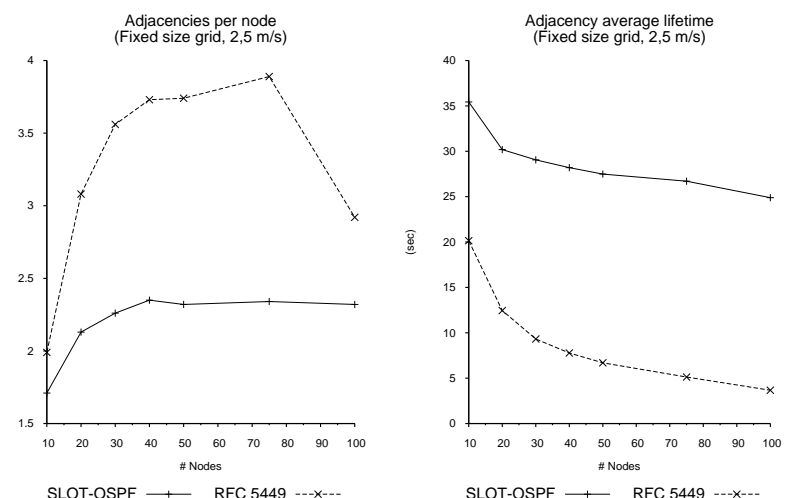

Fig. 4. Average per node number of adjacencies, and adjacency lifetime, in seconds.

We observe in Fig. 2 that while the delivery ratio and the end-to-end delay achieved by SLOT-OSPF remains stable when the number of nodes and the node density in the network grow (we simulated up to 100 nodes), RFC 5449, and then RFC 5614 suffer a significant drop in performance. This drop can be explained by the explosion of the amount of control overhead due to RFC 5449 or RFC 5614 operation.

The following focuses on the comparison between SLOTOSPF and RFC 5449. As shown in Fig. 3, both in terms of sheer size and in terms of number of transmitted control packets, RFC 5449 control traffic increases drastically with the size of the network. The control overhead due to SLOT-OSPF operation grows too with the number of nodes in the network, but at a far more reasonable pace. In fact, the amount of overhead due to RFC 5449 or RFC 5614 operation quickly saturates the available bandwidth and when the density increases as the number of node grows over 75, this saturation makes it more difficult to deliver packets and thus impossible to establish the necessary adjacencies, as shown by the drop in Fig. 4. This makes sense at first sight, as the amount of overhead produced by RFC 5449, for a network with 75 nodes, makes a random node have to bear roughly a local traffic $t_{\text {local }}=\frac{\pi r^{2}}{l^{2}}\left(t_{\text {control }}+t_{\text {data }}\right)$, where $r$ is the radio range $(150 \mathrm{~m}), l$ is the length of the simulated field $(400 \mathrm{~m})$, while $t_{\text {control }}$ is the total control traffic and $t_{\text {data }}$ the total traffic due to user data. In Fig. 3, we measured $t_{\text {control }}$ to be $2,96 \mathrm{Mbps}$, for 75 nodes. In the same context, we measured the average path length to be 1,7 hops. Since the injected data traffic is $1 \mathrm{Mbps}$, this corresponds to $t_{\text {data }}$ being 1,7 Mbps. We therefore get a $t_{\text {local }}$ of approximately 2 Mbps, reaching standard $802.11 \mathrm{~b}$ saturation throughput [21] [22].

\section{Perspectives AND Conclusion}

In this paper, we presented and analyzed the performance of SLOT, a scheme based on Relative Neighborhood Graphs, which provides a backbone overlay over which critical data can be synchronized between neighbors, in a mobile ad hoc context. We have shown that SLOT produces an overlay made of a number of links per node that is independent of the network density and of the topology. We have then applied SLOT to a typical IP protocol which uses such critical data synchronization overlay: the industry-standard routing protocol OSPF, which has recently been extended to work also on mobile ad hoc networks with RFC 5449 or RFC 5614. The simulations show that the use of SLOT-OSPF, a novel OSPF extension for MANETs using SLOT, produces drastically less control traffic than RFC 5449 or RFC 5614. This allows SLOT-OSPF to function correctly as RFC5449 and RFC 5614 stall, when the density and/or number of routers in the domain is large.

We expect however that for real deployments, SLOT with cost based on link quality will perform better for synchronization processes than SLOT with uniform cost. In this context, in order to avoid the $\sqrt{\nu}$ term which can be heavy when the node density increases, we could use a quantized link cost (for 
example $m(A, B)=\lfloor\log \min \{\operatorname{Cost}(A, B), \operatorname{Cost}(B, A)\}\rfloor)$. This would reduce per node synchronization rate, which we expect to be $O(s \log \nu)$. This opens interesting perspectives in the realm of optimized synchronized link overlay.

\section{REFERENCES}

[1] J. Moy: RFC 2328, OSPF Version 2. Internet Society (ISOC). April 1998.

[2] R. Coltun, D. Ferguson, J. Moy: RFC 2740, OSPF for IPv6. Internet Society (ISOC). December 1999.

[3] P. Jacquet, P. Mulhethaler, T. H. Clausen, A. Laouiti, A. Qayyum, L. Viennot: Optimized Link State Routing for Ad Hoc Networks. HIPERCOM Project / INRIA Rocquencourt. Proceedings of the IEEE International Multitopic Conference (INMIC). 2001.

[4] A. Qayyum, L. Viennot, A. Laouiti: Multipoint Relaying for Flooding Broadcast Messages in Mobile Wireless Networks. HIPERCOM Project / INRIA Rocquencourt. Proceedings of the 35th Annual Hawaii International Conference on System Sciencias (HICSS). 2002.

[5] G. F. Riley: The Georgia Tech Network Simulator. Proceedings of the ACM SIGCOMM 2003 Workshops. August 2003.

[6] C. Adjih, E. Baccelli, T. H. Clausen, P. Jacquet, G. Rodolakis: Fish Eye OLSR Scaling Properties. Journal of Communications and Networks. 2003.

[7] T. Henderson, P. Spagnolo, G. Pei: Evaluation of OSPF MANET Extensions. Boeing Technical Report D950-10897-1. July 2005.

[8] P. Jacquet: Optimization of Point-to-point Database Synchronization via Link Overlay RNG in Mobile Ad Hoc Networks, INRIA Research Report RR-6148. February 2007.

[9] E. Baccelli, P. Jacquet, D. Nguyen: Integrating VANETs in the Internet Core with OSPF: the MPR-OSPF Approach. Proceedings of the IEEE International Conference on ITS Telecommunications (ITST). June 2007.

[10] E. Baccelli, T. Clausen, P. Jacquet, D. Nguyen: OSPF Multipoint Relay $(M P R)$ Extension for Ad Hoc Networks. IETF Request For Comments RFC 5449, February 2009.

[11] C. Adjih, E. Baccelli, P. Jacquet: Link State Routing in Ad Hoc Wireless Networks. Proceedings of MILCOM 2003 - IEEE Military Communications Conference, vol. 22, no. 1, pp. 1274-1279, Boston, USA, Oct. 2003.

[12] E. Baccelli, F. Baker, M. Chandra, T. Henderson, J. Macker, R. White: Problem Statement for OSPF Extensions for Mobile Ad Hoc Routing. Internet Engineering Task Force (IETF). draft-baker-manet-ospf-problemstatement-00 (work in progress), 2003.

[13] GNU Zebra, www.zebra.org

[14] INRIA OSPF Extension For MANET Code, www.emmanuelbaccelli.org/ospf

[15] T.M. Cover, P.E. Hart, Nearest Neighbor Pattern Classification, IEEE Transactions on Information Theory, vol. IT-13, No.1, 1967, pp.21-27.

[16] G. Toussaint, The Relative Neighborhood Graph of Finite Planar Set, Pattern Recognition 12 (4), 261268, 1980.

[17] L. Devroye, On The Expected Size of Some Graphs in Computational Geometry, Computers and Mathematics with Applications, vol. 15, pp. 53-64, 1988.

[18] P. Jacquet, Control of mobile ad hoc networks, Proceedings of ITW 2006, Uruguay, 2006.

[19] T. Henderson, P. Spagnolo, J. H. Kim, A Wireless Interface Type for $O S P F$, Proceedings of the IEEE MILCOM Conference, 2003.

[20] E. Baccelli, J.A. Cordero, P. Jacquet, Multi-Point Relaying Techniques with OSPF on Ad Hoc Networks, Proceedings of ICSNC 2009, Porto, Portugal, Sept. 2009.

[21] A. Vasan, A. Udaya Shankar, An Empirical Characterization of Instantaneous Throughput in $802.11 \mathrm{~b}$ WLANs, Department of Computer Science, University of Maryland, www.cs.umd.edu/ shankar/Papers/80211b-profile-1.pdf

[22] F. Calí, M. Conti, E. Gregori, IEEE 802.11 Wireless LAN: Capacity Analysis and Protocol Enhancement, Proceedings of INFOCOM 1998, San Francisco, Mar. 1998.

[23] J. Cartigny, F. Ingelrest and D. Simplot, $R N G$ Relay Subset Flooding Protocols in Mobile Ad-Hoc Networks, Int. J. Found. Comput. Sci., volume 14, 2003

[24] Boeing OSPF MANET Extension Quagga GTNets simulation code, http://hipserver.mct.phantomworks.org/ietf/ospf/, 2008.
[25] E. Baccelli, J. A. Cordero, P. Jacquet, Multi-Point Relaying Techniques with OSPF on Ad Hoc Networks, Proceedings of the IEEE International Conference on Systems and Networks Communications (ICSNC). Porto, Sept. 2009.

[26] J. Wu and H.Li, On calculating connected dominating set for efficient routing in ad hoc wireless networks, Workshop on Discrete Algorithms and Methods for Mobile Computing and Communications 7-14, 1999.

[27] R. Ogier, P. Spagnolo, Mobile Ad Hoc Network (MANET) Extension of OSPF Using Connected Dominating Set (CDS) Flooding, IETF Request For Comments RFC 5614, August 2009.

[28] J. Wu, F. Dai, Efficient Broadcasting with Guaranteed Coverage in Mobile Ad Hoc Networks, in IEEE Transactions on Mobile Computing, 2005.

[29] E. Baccelli, J. A. Cordero, P. Jacquet, Optimization of Critical Data Synchronization via Link Overlay RNG in Mobile Ad Hoc Networks, INRIA Research Report RR-7272, April 2010.

\section{APPENDIX: SimULATION ENVIRONMENT}

The following tables describe the simulation environment parameters, and the parameters specific to the protocols considered in this paper. The $\alpha \in[0,1]$ parameter defines the probability of successful reception function w.r.t. the distance, $\alpha=0.5$ standing for moderately lossy channel. Further characterization of $\alpha$ is available in [7]. Note that, for fairness in the comparison with SLOT-OSPF, the adjacency persistency was disabled in RFC 5449. The code used for RFC 5614 simulations is the code developed by Boeing, available in [24], set with LSAFullness $=1$.

TABLE II

General Simulation Parameters.

\begin{tabular}{|c|c|}
\hline Name & Value \\
\hline \multicolumn{2}{|c|}{ Experiment Statistic Parameters } \\
\hline Seed & 0 \\
\hline Samples/experiment & 20 \\
\hline \multicolumn{2}{|c|}{ Traffic Pattern } \\
\hline Type of traffic & CBR UDP \\
\hline Packet size & 1472 bytes \\
\hline Packet rate & $85 \mathrm{pkts} / \mathrm{sec}$ \\
\hline Traffic rate & $1 \mathrm{Mbps}$ \\
\hline \multicolumn{2}{|r|}{ Scenario } \\
\hline Mobility & Random waypoint model \\
\hline Speed & $v=0,5,10,15 \frac{\mathrm{m}}{\mathrm{s}}$ (constant) \\
\hline Grid shape and size & Square, $400 \mathrm{~m} \times 400 \mathrm{~m}$ \\
\hline Radio range & $150 \mathrm{~m}$ \\
\hline Wireless $\alpha$ & 0.5 \\
\hline Pause time & $40 \mathrm{sec}$ \\
\hline MAC protocol & IEEE $802.11 \mathrm{~b}$ \\
\hline \multicolumn{2}{|c|}{ OSPF General Configuration } \\
\hline HelloInterval & $2 \mathrm{sec}$ \\
\hline DeadInterval & $6 \mathrm{sec}$ \\
\hline RxmtInterval & $5 \mathrm{sec}$ \\
\hline MinLSInterval & $5 \mathrm{sec}$ \\
\hline MinLSArrival & $1 \mathrm{sec}$ \\
\hline
\end{tabular}

TABLE III

RFC 5449 AND SLOT-OSPF PARAMETERS.

\begin{tabular}{|c|c|}
\hline Name & Value \\
\hline \hline AckInterval & $1800 \mathrm{msec}$ \\
\hline Flooding MPR? & Yes \\
\hline Topology Reduction & MPR Topology Reduction \\
\hline Adjacency Selection & MPR or SLOT Adjacency Reduction \\
\hline Adjacency Persistency & Disabled \\
\hline \hline
\end{tabular}

